\title{
ACRL members running for ALA Council in the spring 2018 election
}

The following ACRL members are either nominated or petition candidates for ALA councilor. ACRL members are encouraged to vote for these candidates to increase ACRL's voice in ALA affairs.

Emily Bergman, Adjunct Reference Librarian, Glendale (California) Community College

Whitney Buccicone, Special Collections Cataloging Librarian, University of Washington, Seattle, Washington

Roberto C. Delgadillo, Research Support Services Librarian, Peter J. Shields Library, University of California-Davis

Megan Hodge, Teaching and Learning Librarian, Virginia Commonwealth University, Richmond, Virginia

Kathy M. Irwin, Associate Dean of Libraries, Central Michigan University, Mount Pleasant, Michigan

Susan L. Jennings, Dean of Library Services, Chattanooga State Community College, Chattanooga, Tennessee

kYmberly Keeton, Solo Academic Librarian, ART_library deco, Jefferson City, Missouri

Rebekah Kilzer, Director, Member Education, OCLC, Dublin, Ohio
Linda A. Kopecky, Head, Research Services, University of Wisconsin-Milwaukee

Leo S. Lo, Associate University Librarian, Old Dominion University, Norfolk, Virginia

Steve Matthews, Librarian (emeritus), Foxcroft School, Middleburg, Virginia

Joe Mocnik, Dean of Libraries, North Dakota State University, Fargo, North Dakota

Toni Negro, Librarian, University of Maryland, Priddy Library, Rockville, Maryland

Alexandra P. Rivera, Student Success and Community Engagement Librarian, University of Michigan Library, Ann Arbor, Michigan

Jim Teliha, Director of the Library and Learning Commons, Utica College, Utica, New York

Erica Ann Watson, Librarian, Art Institute of California, San Francisco, California

Joan S. Weeks, Head, Near East Section and Turkish Specialist, Library of Congress, African and Middle Eastern Division, Washington, D.C.

Janice Welburn, Dean of Libraries, Marquette University, Milwaukee, Wisconsin

Donald Welch, Manager, Learning Resource Center, Fortis College, Grand Prairie, Texas 